\title{
Digest Review of Yoga as an Effective Treatment for those with Low Back Pain
}

\author{
Johnston Green A* \\ Department of Health \& Human Performance, Tarleton State University, USA
}

*Corresponding author: Department of Health and Human Performance, Tarleton State University, USA.

Received Date: October 29, 2019

Published Date: December 09, 2019

\section{Clinical Question}

Is yoga a more effective treatment than standardized care for those over 18 years of age with low back pain?

\section{Data Sources}

Studies were identified by searching Medline, the Cochrane Library, Embase, Cambase, and Psycinfo databases through January 2012. The search included key terms yoga and low back pain with specific adaptations as needed on a database by database basis. References in each of the articles were also screened.

\section{Study Selection}

Articles included in this systematic review were randomized controlled trials that compared yoga with any other intervention for participants older than $18 \mathrm{y}$ who had low back pain regardless of etiology. Each article also employed at least 1 patient-centered outcome measure.

\section{Quality Assessment and Data Extraction}

Independently, two reviewers appraised the caliber and composition of each article while employing a third review if needed to reach an agreement in case of disparity in evaluation results. The 12 criteria suggested by the Cochrane Back Review Group in the 2009 Updated Method Guidelines for Systematic Reviews were employed by the reviewers. Each item was rated as yes, no, or unclear. To be designated as having low risk of bias, a study had to achieve at least 6 of 12 criteria without possessing a serious flaw. Outcome assessment was determined to be shortterm if performed close to 12 weeks post-intervention. Long-term assessment was deemed to be follow-up close to 12 months after the treatment. This systematic review had the reviewers extract data which included participant characteristics, control conditions, interventions, co-interventions, outcome measures, and results.

\section{Main Results}

Originally 160 records were identified from the search of the databases. After removing duplicate articles and excluding those that did not meet criteria, 12 full-text articles were assessed for quality with 10 selected for the qualitative systematic review and 8 of those being included in the quantitative meta-analysis. As assessed using the 12 criteria in the 2009 Updated Method Guidelines for Systematic Reviews as established by the Cochrane Back Review Group, eight studies showed a low risk of bias with two studies having a high risk of bias.

The results of the studies were split into the short-term and long-term categories. These two categories were assessed for the each of the following three effects as applicable:

- Pain.

- $\quad$ Back-specific disability.

- Health-related quality of life.

The recommendations of the Cochrane Back Review Group provided for the valuation of the levels of evidence for each study. Strong evidence was found for the short-term effectiveness on pain (standardized mean differences (SMD) $=-0.48 ; 95 \% \mathrm{CI},-0.65$ to $-0.31 ; \mathrm{P}<0.01$ ) and back -specific disability (SMD $=-0.59 ; 95 \%$ CI, -0.87 to -0.30 ; $\mathrm{P}<0.01$ ). Long term benefits were supported by moderate evidence in terms of pain (SMD $=-0.33 ; 95 \% \mathrm{CI},-0.59$ to $-0.07 ; \mathrm{P}=0.01$ ) and back-specific disability (SMD $=-0.35 ; 95 \% \mathrm{CI}$, -0.55 to -0.15 ; $\mathrm{P}<0.01$ ). Effectiveness was assessed by patientcentered outcomes for pain and back-specific disability. Quality of life was also evaluated with no evidence reported at either the short or the long term. 


\section{Conclusion}

There is currently limited data available which makes it challenging to designate yoga as either superior or inferior to the standard level of treatment for LBP. However, the data presented in this systematic review suggests that yoga does not provide for detrimental effects and lends itself to be more effective than education alone. A prior qualitative systematic review from Posadzki P and Ernst E [1] along with Büssing A, et al. [2] metaanalysis on yoga for chronic pain2have reached similar conclusions with encouraging evidence that yoga is an applicable treatment for LBP. Additionally, there is promise of two new large-scale RCTs providing supporting data.

\section{Commentary}

Low back pain (LBP) is a frequent and often high cost reason for health care provider visits in the United States. [3] As such, it is crucial to find effective and low-cost treatment options for those that suffer from this condition. LBP may be diagnosed and treated based on symptom presentation including radiculopathy, musculoskeletal tissue abnormalities, and the cause for the dysfunction [3]. Exercise has been determined to be an effective treatment for LBP with yoga being included in this intervention [4]. However, the level of effect that yoga included exercise has on LBP are small.

Studies have been performed which compared the benefits of yoga, exercise, and education as therapeutic interventions for those with LBP. While not always noted, various approaches to yoga therapy studied included integrative techniques, Viniyoga, Hatha, and Iyengar. A study in this systematic review contrasted the use of Viniyoga to an exercise class targeted toward those with LBP as well as the use of a back-pain care book for education [4]. The Viniyoga and exercise class were each performed for 75 minutes on a weekly basis [4]. The mainly middle-aged population (30-40 years of age) of this study reported that the Viniyoga produced positive results lasting 14 weeks after the intervention's conclusion in regard to pain and back-specific disability as assessed by a modified Roland Disability Scale and the Short-Form 36 Health Survey [4]. These outcomes were in comparison to the exercise group which had some improvement while the book group reporting very minimal decreases in disability [4]. While there has yet to be published evidence of the mechanisms by which yoga is able to ease pain, the concept of yoga is that it teaches one to unite their body, mind, and soul through a psycho-somatic-spiritual connection during its practice [5]. Additional support of the benefits of this practice were discovered when comparing Iyengar yoga with education for the treatment of chronic low back pain (CLBP) almost a decade later. [6] This randomized control trial performed by Williams, et al. [6] assessed Iyengar and educational interventions in patients with LBP symptomology lasting longer than 3 months by splitting them into a group per each treatment. Iyengar yoga places an emphasis on structural alignment using props in conjunction with a specific sequence of poses and breath control [6]. Much like the other studies, the yoga group showed significant improvement in pain level and management as well as standard of disability not only at the end of the intervention but at a 3-month follow-up when compared to the education only group [6].

While multiple studies have shown the advantages to using yoga for LBP when compared to standardized treatment, it is important to note that not each practice discipline will produce the same results. Kundalini yoga which can be performed seated, lying, or standing and lends itself to be more gentle than traditional forms of yoga was compared to strength training as well as education in regard to efficacy as a treatment for back pain [7]. Based on the results of those that practiced Kundalini once a week, there were no statistically significant difference when compared with attending a weekly strength training class in regard to missing work due to pain and disability [7-8].

This particular systematic review revealed yoga to be an appropriate treatment approach when addressing those with LBP following a minimum of 75 minute session once a week with additional practice homework of 30 minutes 3-4 times a week Further support for the use of specific disciplines of yoga in those with LBP compared with exercise and/or education is evident when studies are assessed on an individual basis. The concept that yoga does not lend itself to many unfavorable effects when practiced by those with LBP makes it attractive as a treatment avenue especially for active patients. Continued research is needed at this time to ascertain the connection between yoga and its ability to improve pain levels and function in those with LBP.

\section{Acknowledgment}

None.

\section{Conflict of Interest}

No conflict of interest.

\section{References}

1. Posadzki P, Ernst E (2011) Yoga for low back pain: a systematic review of randomized clinical trials. Clin Rheumatol 30(9): 1257-1262.

2. Büssing A, Ostermann T, Lüdtke R (2012) Effects of yoga interventions on pain and pain associated disability: a meta-analysis. J Pain 13(1): 1-9.

3. Qaseem A, Wilt TJ, Mclean RM, Forciea MA (2017) Noninvasive Treatments for Acute, Subacute, and Chronic Low Back Pain: A Clinical Practice Guideline from the American College of Physicians. Ann Intern Med 166(7): 514-530.

4. Sherman KJ, Cherkin DC, Erro J, Miglioretti DL, Deyo RA (2005) Comparing yoga, exercise, and a self-care book for chronic low back pain: a randomized, controlled trial. Ann Intern Med 143(12): 849-56.

5. Balaji PA, Varne SR, Ali SS (2012) Physiological effects of yogic practices and transcendental meditation in health and disease. N Am J Med Sci 4(10): 442-448

6. Williams KA, Petronis J, Smith D (2005) Effect of Iyengar yoga therapy for chronic low back pain. Pain 115(1-2): 107-117.

7. Brämberg EB, Bergström G, Jensen I, Hagberg J, Kwak L (2017) Effects of yoga, strength training and advice on back pain: a randomized controlled trial. BMC Musculoskelet Disord 18(1): 132.

8. Cramer H, Lauche R, Haller H, Dobos G (2013) A Systematic Review and Meta-Analysis of Yoga for Low Back Pain. Clin J Pain 29(5): 450-460. 